\title{
Ingolf Max
}

\section{EXISTENCE, \\ the Square of Opposites, and Two-Dimensional Logic}

\author{
CONTENTS
}

\author{
1. The Starting Point \\ 2. The Two-Dimensional System $\mathcal{Q}$ \\ 3. A New Representation of the Square of Opposites \\ 4. Outlook: Variable Quantifiers
}




\section{The Starting Point}

Ontological commitments and other problems concerning existence arise in connection with various aspects of logical theories. The semantics of quantification theory is usually formulated in such a manner that theorems are all and only those formulae which come out true under all interpretations in all non-empty domains. There are several approaches to include the empty domain. Paradoxically this apparent semantic extension means surrendering several formulae which are valid and intuitively plausible.

Outside of these questions concerning general existence there are a lot of difficulties regarding singular existence: Is it a tacit presupposition that any singular term has a meaning/denotes an entity (Frege)? Does quantifying singular terms commit us to acknowledge singular existence (Quine)? Is Existence a first order predicate, a predicate at all? If so, how is it do define? $\exists ! x \stackrel{\text { df }}{=} \exists x F x$ or $\exists ! x \stackrel{\text { df }}{=} \exists x(x=a)$ or $x=x$ ?

In this paper I am going to discuss questions of existence connected with the traditional square of opposites. There are several approaches to explicate the logical connection. But solutions, if any, are heterogeneous, incomplete or do not take into consideration the empty domain. After introducing my two-dimensional system $\mathcal{Q}$ I want will demonstrate the expressive power of such a framework by offering a new explication of the square of opposites. Two-dimensionality allows the syntactical formalization of existence conditions explicitly. Negation can be understood as denying only the explicitly asserted but not the implicitly presumed (existence) part. Regarding categorical inferences both assertion and existence presupposition can be relevant. A unique two-dimensional translation of the traditional square of opposites covers all interesting cases.

\section{The Two-Dimensional System $\mathcal{Q}$}

In this section I introduce the formal background for my discussion of several two-dimensional ways of representing existence and some interesting variable quantifiers.

\subsection{Primitive symbols}

1. Propositional variables: $p, q, r, s, p_{1}, \ldots$

2. Classical functors: $\sim, \wedge, \vee, \supset, \equiv, \not \equiv$. 
3. Individual variables: $x, y, z, x_{1}, \ldots$

4. Individual constants: $a, b, c, a_{1}, \ldots$

5. Infinite list of singular, binary etc. functional variables: $F^{1}, G^{1}, H^{1}$, $F_{1}^{1}, \ldots, F^{2}, G^{2}, H^{2}, F_{1}^{2}, \ldots$

6. Quantifiers: $\forall, \exists$

7. Equality: $=$.

8. Operator forming pairs of classical formulae: [ ].

9. Form of 1-placed, 2-placed variable functors: $V_{i}^{1}(1 \leqslant i \leqslant 4)$, $V_{j}^{2}\left(1 \leqslant j \leqslant 4^{4^{n}}\right)$.

10. Form of variable quantifiers: $\forall_{i}, \exists_{j}$.

11. Parentheses: ( ) ( ).

\subsection{Formation rules}

1. A propositional variable standing alone is a formula of $\mathcal{Q}$.

2. If $\boldsymbol{f}$ is an $n$-ary functional variable and if $\boldsymbol{a}_{1}, \boldsymbol{a}_{2}, \ldots, \boldsymbol{a}_{n}$ are individual variables or individual constants or both (not necessarily all different), then $\boldsymbol{f}\left(\boldsymbol{a}_{1}, \boldsymbol{a}_{2}, \ldots, \boldsymbol{a}_{n}\right)$ and $\boldsymbol{a}_{1}=\boldsymbol{a}_{2}$ are formulae of $\mathcal{Q}$.

3. If $X$ and $Y$ are formulae of $\mathcal{Q}$, then $\sim X,(X \wedge Y),(X \vee Y),(X \supset Y)$, $(X \equiv Y)$ and $(X \not \equiv Y)$ are formulae of $\mathcal{Q}$.

4. If $X$ is a formula of $\mathcal{Q}$ and $\boldsymbol{a}$ is an individual variable, then $\forall \boldsymbol{x} X$ and $\exists \boldsymbol{x} X$ are formulae of $\mathcal{Q}$.

5. If $A, B$ are formulae of $\mathcal{Q}$ formed without reference to the formation rules 5.-7. (i.e. usual classical formulae), then $\left[\begin{array}{l}A \\ B\end{array}\right]$ is a formula of $\mathcal{Q}$.

6. If $X, Y$ are formulae of $\mathcal{Q}$, then $V^{1} X$, and $V^{2} X Y$ are formulae of $\mathcal{Q}$.

7. If $X$ is a formula of $\mathcal{Q}$ and $\boldsymbol{x}$ is an individual variable, then $\forall_{i} \boldsymbol{x} X$ and $\exists_{j} \boldsymbol{x} X$ are formulae of $\mathcal{Q}$.

8. $X$ is a formula of $\mathcal{Q}$ iff its being so follows from 1.-7.

\subsection{Types of formulae}

CL-Formulae $A, B, C, D$ (i.e. classical formulae) are those formulae which were exclusively formed by means of formation rules $1 .-4$.

An E-Formula $\mathcal{E}$ (i.e. elementary formula) is a formula of the form $\left[\begin{array}{l}A \\ B\end{array}\right]$.

F-FORMULAE $\mathcal{F}$ are formulae of the forms: $\quad V_{i}^{1}\left[\begin{array}{l}A \\ B\end{array}\right], \quad V_{i}^{1} A, \quad V_{j}^{2}\left[\begin{array}{l}A \\ B\end{array}\right]\left[\begin{array}{l}C \\ D\end{array}\right]$, $V_{j}^{2}\left[\begin{array}{l}A \\ B\end{array}\right] C, \quad V_{j}^{2} A\left[\begin{array}{l}B \\ C\end{array}\right], \quad V_{j}^{2} A B, \quad \forall_{i} \boldsymbol{x}\left[\begin{array}{l}A \\ B\end{array}\right], \quad \exists_{j} \boldsymbol{x}\left[\begin{array}{l}A \\ B\end{array}\right]$. 
An NC-Formula $\mathcal{Z}$ (i.e. a non-classical formula) is a formula of $\mathcal{Q}$ which is neither a CL-formula nor an E-formula.

\subsection{Reduction rules}

How should reduction rules act in the system $\mathcal{Q}$ ? Roughly speaking, reduction rules should support a complete transformation of any non-classical formula $\mathcal{Z}$ to a formula of the form $\left[\begin{array}{l}A \\ B\end{array}\right]$ (i.e. an E-formula of a special kind).

I use the following abbreviation of $X \Longrightarrow X\left[Y_{1} / Y_{2}\right]: Y_{1} \Longrightarrow Y_{2}$. Both " $X \Longrightarrow X\left[Y_{1} / Y_{2}\right]$ " and " $Y_{1} \Longrightarrow Y_{2}$ " are read as "From $X$ to infer $X\left[Y_{1} / Y_{2}\right]$ ", with $X\left[Y_{1} / Y_{2}\right]$ we mean that formula which is the result of substituting any formula $Y_{2}$ for the formula $Y_{1}$ in all of its occurrences in $X$.

1. Reduction rules for classical functors and quantifiers:

(i) $\sim\left[\begin{array}{l}A \\ B\end{array}\right] \Longrightarrow\left[\begin{array}{l}\sim A \\ \sim B\end{array}\right]$

(ii) $\left[\begin{array}{l}A \\ B\end{array}\right] \wedge\left[\begin{array}{l}C \\ D\end{array}\right] \Longrightarrow\left[\begin{array}{l}A \wedge C \\ B \wedge D\end{array}\right]$

$$
\left[\begin{array}{l}
A \\
B
\end{array}\right] \wedge C \Longrightarrow\left[\begin{array}{l}
A \wedge C \\
B \wedge C
\end{array}\right] \quad A \wedge\left[\begin{array}{l}
B \\
C
\end{array}\right] \Longrightarrow\left[\begin{array}{l}
A \wedge B \\
A \wedge C
\end{array}\right]
$$

(iii) Disjunction, implication, equivalence, and negequivalence (exclusive disjunction) as in 1.2.

(iv) $\forall \boldsymbol{x}\left[\begin{array}{l}A \\ B\end{array}\right] \Longrightarrow\left[\begin{array}{l}\forall \boldsymbol{x} A \\ \forall \boldsymbol{x} B\end{array}\right] \quad \exists \boldsymbol{x}\left[\begin{array}{l}A \\ B\end{array}\right] \Longrightarrow\left[\begin{array}{l}\exists \boldsymbol{x} A \\ \exists \boldsymbol{x} B\end{array}\right]$

2. Reduction rules for variable functors

The general form of substitution is

$S R$

$$
X \Longrightarrow X[\mathcal{F} / \mathcal{E}]
$$

where by $X[\mathcal{F} / \mathcal{E}]$ I mean the result of substituting the $E$-formula $\mathcal{E}$ for the $F$-formula $\mathcal{F}$ in all occurrences of $\mathcal{F}$ in $X$.

The special forms of V-SUbSTitution are

(i) $V^{1}\left[\begin{array}{l}A \\ B\end{array}\right] \Longrightarrow\left[\begin{array}{l}\Phi^{2} A B \\ \Psi^{2} A B\end{array}\right]$ 
(ii) $V^{1} A \Longrightarrow\left[\begin{array}{l}\Phi^{2} A A \\ \Psi^{2} A A\end{array}\right]$

(iii) $V^{2}\left[\begin{array}{l}A \\ B\end{array}\right]\left[\begin{array}{l}C \\ D\end{array}\right] \Longrightarrow\left[\begin{array}{l}\Phi^{4} A B C D \\ \Psi^{4} A B C D\end{array}\right]$

(iv) $V^{2}\left[\begin{array}{l}A \\ B\end{array}\right] C \Longrightarrow\left[\begin{array}{l}\Phi^{4} A B C C \\ \Psi^{4} A B C C\end{array}\right]$

(v) $V^{2} A\left[\begin{array}{l}B \\ C\end{array}\right] \Longrightarrow\left[\begin{array}{l}\Phi^{4} A A B C \\ \Psi^{4} A A B C\end{array}\right]$

(vi) $V^{2} A B \Longrightarrow\left[\begin{array}{l}\Phi^{4} A A B B \\ \Psi^{4} A A B B\end{array}\right]$

where $\Phi^{2}, \Psi^{2}$ are 2-placed classical functors, and $\Phi^{4}, \Psi^{4}$ are 4-placed classical functors definable by given functors (because of the truth-functional completeness of classical logic). It is immediately clear that V-substitution 2(ii) is a subcase of 2(i), and V-substitutions 2(iv), 2(v), and 2(vi) are subcases of 2 (iii).

3. Reduction rules for variable quantifiers

The special forms of Q-SUBSTITUTION are

(i) $\forall_{i} \boldsymbol{x}\left[\begin{array}{l}A \\ B\end{array}\right] \Longrightarrow\left[\begin{array}{l}\forall \boldsymbol{x} \Phi^{2} A B \\ \forall \boldsymbol{x} \Psi^{2} A B\end{array}\right] \quad \forall i \boldsymbol{x} A \Longrightarrow\left[\begin{array}{l}\forall \boldsymbol{x} \Phi^{2} A A \\ \forall \boldsymbol{x} \Psi^{2} A A\end{array}\right]$

(ii) $\exists_{i} \boldsymbol{x}\left[\begin{array}{l}A \\ B\end{array}\right] \Longrightarrow\left[\begin{array}{l}\exists \boldsymbol{x} \Phi^{2} A B \\ \exists \boldsymbol{x} \Psi^{2} A B\end{array}\right] \quad \exists_{i} \boldsymbol{x} A \Longrightarrow\left[\begin{array}{l}\exists \boldsymbol{x} \Phi^{2} A A \\ \exists \boldsymbol{x} \Psi^{2} A A\end{array}\right]$

Later special V-REDUCTION and Q-REDUCTION rules will be formulated in such a way, that every NC-FORMULA can be transformed into an E-formula in a finite number of steps starting from the inside of a given NC-formula.

Example: Let $\neg$ be a variable functor and $\forall_{1}$ be a variable quantifier characterized by the following reduction rules

$$
\neg\left[\begin{array}{l}
A \\
B
\end{array}\right] \Longrightarrow\left[\begin{array}{c}
\sim A \\
B
\end{array}\right] \quad \forall_{1} \boldsymbol{x}\left[\begin{array}{l}
A \\
B
\end{array}\right] \Longrightarrow\left[\begin{array}{l}
\forall \boldsymbol{x}(B \supset A) \\
\forall \boldsymbol{x}(B \supset A)
\end{array}\right]
$$

and suppose the following NC-formula is given:

$$
\neg \neg \forall_{1} x\left[\begin{array}{c}
G x \\
F x
\end{array}\right] \equiv \forall x(F x \supset G x) .
$$


Then we can definitely generate a corresponding E-formula using the following steps:

$$
\begin{aligned}
& \neg \neg\left[\begin{array}{c}
\forall x(F x \supset G x) \\
\forall x(F x \supset G x)
\end{array}\right] \equiv \forall x(F x \supset G x) \\
& \neg\left[\begin{array}{c}
\sim \forall x(F x \supset G x) \\
\forall x(F x \supset G x)
\end{array}\right] \equiv \forall x(F x \supset G x) \\
& {\left[\begin{array}{c}
\sim \sim \forall x(F x \supset G x) \\
\forall x(F x \supset G x)
\end{array}\right] \equiv \forall x(F x \supset G x)} \\
& {\left[\begin{array}{c}
\sim \sim \forall x(F x \supset G x) \equiv \forall x(F x \supset G x) \\
\forall x(F x \supset G x) \equiv \forall x(F x \supset G x)
\end{array}\right]}
\end{aligned}
$$

\subsection{Semantics}

Validity and inconsistency of CL-formulae. Let $\vdash A$ indicate that the classical formula $A$ is CLASSICALLY VALID (is a TAUTOLOGY), i.e. valid in the usual classical sense.

Furthermore, let $\nvdash A$ indicate that the classical formula $A$ is NOT CLASSICALLY VALID (is NOT A TAUTOLOGY).

\section{Validity of E-formulae.}

Definition 1. The E-formula $\left[\begin{array}{l}A \\ B\end{array}\right]$ is $\boldsymbol{E}$-valid (symb.: $\models\left[\begin{array}{l}A \\ B\end{array}\right]$ ) iff $\vdash A$ and $\vdash B$.

Theorem 1. $\models\left[\begin{array}{l}A \\ B\end{array}\right]$ iff $\vdash(A \wedge B)$.

Since all $N C$-formulae can be reduced to $E$-formulae this theorem means that validity in $\mathcal{Q}$ is reducible to classical validity. Hence, what follows is the representation of existence in a classical style.

\section{Validity of NC-formulae}

Definition 2. Let $\mathcal{Z}$ be any $N C$-formula and $\left[\begin{array}{l}A_{\mathcal{Z}} \\ B_{\mathcal{Z}}\end{array}\right]$ that E-formula which is the result of the complete reduction of $\mathcal{Z}$, i.e. that both all occurrences of variable functors and all occurrences of classical functors outside the scope of brackets are eliminated:

$$
\models \mathcal{Z} \quad \text { iff } \quad \models\left[\begin{array}{l}
A_{\mathcal{Z}} \\
B_{\mathcal{Z}}
\end{array}\right]
$$




\section{A New Representation of the Square of Opposites}

It has been widely discussed that there is apparently no perfect formal representation of the traditional square of opposition by using modern formal translations. Which circumstances are responsible for this situation?

\subsection{The traditional square of opposites}

Let us begin with a short characterization of the traditional situation: Usually propositions not compounded of other propositions are called categorical. They have a subject term and a predicate term. In the famous example "All men are mortal", "men" is the subject term and "mortal" the predicate term. According to their QUANTITY, categorials can be subdivided into universals ("All men are mortal", "No men are mortal") and particulars ("Some men are mortal"), and according to their QUALITY, into affirmatives ("All men are mortal", "Some men are mortal") and negatives ("No men are mortal", Some men are not mortal").

The traditional four forms of categorial propositions are the following:

A-propositions E-propositions universal affirmatives universal negatives I-propositions particular affirmatives O-propositions
All $F$ 's are $G$ 's All F's are not $G$ 's Some $F$ 's are $G$ 's Some $F$ 's are not $G$ 's

The traditional name square of opposites goes back to the fact that propositions with the same terms in the same order may be opposed in several ways:

(a) $\quad \mathbf{A}$ and $\mathbf{O}$ are contradictories (cannot be jointly true or jointly false)

(b) $\quad \mathbf{E}$ and $\mathbf{I}$ are contradictories

(c) $\quad \mathbf{A}$ and $\mathbf{E}$ are contraries (cannot be jointly true)

(d) $\quad \mathbf{I}$ and $\mathbf{O}$ are subcontraries (cannot be jointly false)

(e) $\quad \mathbf{A}$ and $\mathbf{I}$ are subalterns

(f) $\quad \mathbf{E}$ and $\mathbf{O}$ are subalterns

$$
\begin{aligned}
& \mathbf{A} \dashv \sim \mathbf{O} \\
& \mathbf{E} \dashv \vdash \sim \mathbf{I} \\
& \vdash \sim(\mathbf{A} \wedge \mathbf{E}), \mathbf{A} \vdash \sim \mathbf{E} \\
& \vdash \mathbf{I} \vee \mathbf{O}, \sim \mathbf{I} \vdash \mathbf{O}
\end{aligned}
$$

$$
\begin{aligned}
& \mathbf{A} \vdash \mathbf{I} \text { and } \mathbf{I} \nvdash \mathbf{A} \\
& \mathbf{E} \vdash \mathbf{O} \text { and } \mathbf{O} \nvdash \mathbf{E}
\end{aligned}
$$


If we display this situation as a rectangle - with universals at the top, particulars at the bottom, affirmatives on the left, negatives on the right we get the following picture:

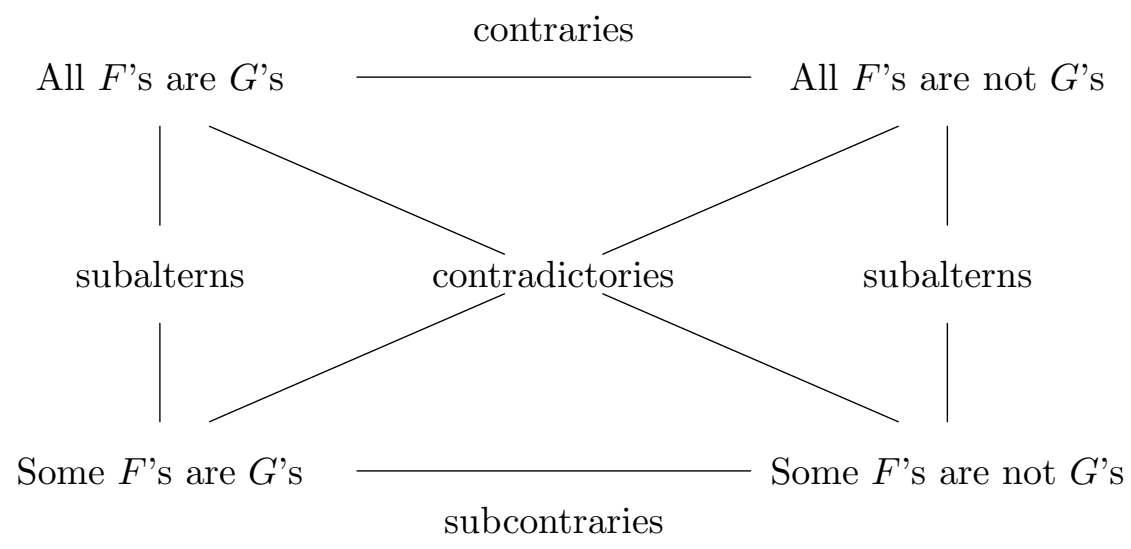

\subsection{Modern translations}

Let us try the usual translation into a Russellian formalism:
A: $\forall x(F x \supset G x)$
E: $\forall x(F x \supset \sim G x)$
I: $\quad \exists x(F x \wedge G x)$
O: $\exists x(F x \wedge \sim G x)$

With respect to this translation (a) and (b) are valid, but (c) to (f) are not valid. ${ }^{1}$

Leonard (cf. [4], 51) argues that the modern, classical logic is rich enough to explicate the presupposition. ${ }^{2}$ Hence, we have to modify the translation for A- and E-expressions:
A: $\forall x(F x \supset G x) \wedge \exists x F x$
E: $\forall x(F x \supset \sim G x) \wedge \exists x F x$.

\footnotetext{
${ }^{1}$ (a) and (b) are valid including the emty domain, whereas (c) to (f) are not valid even in all non-empty domains.

2 Following Frege's ([1], 40) terminology of "selbstverständliche Voraussetzung" with respect to singular terms Leonard uses the notion "tacit, unexpressed presupposition" with respect to general terms.
} 
But now we are faced with a dilemma: on the one hand, we get the result that (c) to (f) are valid under this circumstances, on the other, we loose the validity of (a) and (b) and, therefore, the definability of one quantifier by using the other. Leonard argues that this two-faced solution is a "real advance":

Must we say that the traditional logic was in error? Not at all! On the contrary, it may be held that the traditional logic was a quite correct abstract system of logic; but that it was set up and developed with a tacit, or unexpressed presupposition: namely, that its terms, S, $\mathrm{P}$, etc., were terms having existent exemplars.

At the same time, the modern logic marks a real advance. With its symbolism for quantifiers, it can represent the universal presupposition of the traditional logic. Hence, it can express its relevance when it is relevant (as in inference from $\mathbf{A}$ to $\mathbf{I}$, express irrelevance (by omitting mention of it) when it is irrelevant (as in the inference from $\mathbf{A}$ to not $\sim \mathbf{O}$ and even explore the consequence of its falsity. This gives the modern logic a much wider applicability then that enjoyed by the traditional logic. ([4], 51)

It remains an unsolved puzzle to give a unique translation corresponding to the whole square of opposites. If we take a universe of discourse (the $F$ 's) for granted we are able to offer an apparently simple solution:
A: $\forall x G x$
$\mathbf{E}: \forall x \sim G x$
I: $\exists x G x$
O: $\exists x \sim G x$

It is easy to check the validity of (a) to (f) in the non-empty domains. Quine, among others (e.g. [6], [2] and [3]), offers an axomatization of the first-order predicate calculus called inclusive quantification theory, i.e. inclusive of the empty domain. But there is an easy test regarding the empty domain:

An easy supplementary test enables us anyway, when we please to decide whether a formula holds for the emty domain. We have only to mark the universal quantifications as true and the existential ones as false, and apply truth-table considerations. ([7], 177)

Using this "supplementary test" we have to acknowledge that our last proposal does not work for (c) to (f) in the empty domain.

Strawson ([8], 170f.) offers a "formalistic solution" which is somehow crazy and gives cause for searching for a better "realistic solution" which "illuminates some general features of our ordinary speech" $([8], 170)$. Here is Strawson's "formalistic solution" without further comment: 

A: $\forall x(F x \supset G x) \wedge \exists x F x \wedge \exists x \sim G x$
E: $\quad \forall x(F x \supset \sim G x) \wedge \exists x F x \wedge \exists x G x$
I: $\quad \exists x(F x \wedge G x) \vee \exists x F x \vee \sim \exists x G x$
E: $\exists x(F x \wedge \sim G x) \vee \exists x F x \vee \exists x \sim G x$.

Any tested solution seems to force us to the following alternative: either to take existential preconditions for granted (tacit presuppositions), i.e. there is no need for a syntactic or even semantic representation of such preconditions, or to find a syntactic place and formalization of existence which seems to be possible only by adding a conjunct: . . $\wedge \exists F x .{ }^{3}$ But using logical conjunction forces us to give up the intuitive difference between explicit (asserted) and implicit (presupposed) meaning.

My thesis is that a two-dimensional framework allows to leave this pseudo-forcing alternative. I offer a unifying syntactic approach without conjunction at the beginning. The empty domain is also taken into consideration. But how does it work?

\subsection{A two-dimensional representation of the square of opposites}

I interpret the $A$ in the E-expression $\left[\begin{array}{l}A \\ B\end{array}\right]$ as ASsertion, and the $B$ as PRESUPPOSITION without further qualification.

PRESUPPOSITION-PRESERVING/INTERNAL NEGATION: $\neg\left[\begin{array}{l}A \\ B\end{array}\right] \Longrightarrow\left[\begin{array}{c}\sim A \\ B\end{array}\right]$

Given this situation it is useful to introduce the following pseudo 3-valued reading of E-expressions:

1. $\left[\begin{array}{l}A \\ B\end{array}\right]=$ TRUE iff $A=1$ and $B=1$,

2. $\left[\begin{array}{l}A \\ B\end{array}\right]=$ FALSE $\quad$ iff $A=0$ and $B=1$,

3. $\left[\begin{array}{l}A \\ B\end{array}\right]=$ INCORRECT iff $B=0$.

Now it is clear that we should try the following translation of categorical expressions:

$\mathbf{A}:\left[\begin{array}{c}\forall x(F x \supset G x) \\ \exists x F x\end{array}\right]$

${ }^{3}$ Other candidates discussed in the literature are $x=x$ and $\exists x(x=a)$. 
$\begin{array}{ll}\text { E: } & {\left[\begin{array}{c}\forall x(F x \supset \sim G x) \\ \exists x F x\end{array}\right]} \\ \text { I: } & {\left[\begin{array}{c}\exists x(F x \wedge G x) \\ \exists x F x\end{array}\right]} \\ \text { O: } & {\left[\begin{array}{c}\exists x(F x \wedge \sim G x) \\ \exists x F x\end{array}\right] .}\end{array}$

It is sufficient to explicate existence by using $\exists x F x$ in any of the four cases (the $B$-part of E-expressions). The assertion part is identical with the Russellian translation above. But there is no conjunction-connection between assertion/first line and existence presupposition/second line.

The next step consists in interpreting any negation whose argument contains E-expressions as PRESUPPOSITION-PRESERVING/INTERNAL negations. This is in accordance with the idea that presuppositions are normally invariant regarding negation.

Finally, I introduce a variable functor with the following reduction property:

$$
\left[\begin{array}{l}
A \\
B
\end{array}\right] \rightarrow\left[\begin{array}{l}
C \\
D
\end{array}\right] \Longrightarrow\left[\begin{array}{l}
A \wedge B \supset C \\
A \wedge B \supset D
\end{array}\right]
$$

We can read the expression " $=X \rightarrow Y$ " as "From $X$ follows $Y$ ". Inference in this sense allows the inclusion of implicit/presupposed premisses explicitly. Combining these points we obtain a re-formulation of the logical properties of the square of opposites:

(a) $\mathbf{A}$ and $\mathbf{O}$ are contradictories

$$
\begin{aligned}
& \models \mathbf{A} \rightarrow \neg \mathbf{O} \text { and } \models \neg \mathbf{O} \rightarrow \mathbf{A} \\
& \models \mathbf{E} \rightarrow \neg \mathbf{I} \text { and } \models \neg \mathbf{I} \rightarrow \mathbf{E} \\
& \models \mathbf{A} \rightarrow \neg \mathbf{E}) \\
& \models \neg \mathbf{I} \rightarrow \mathbf{O} \\
& \models \mathbf{A} \rightarrow \mathbf{I} \text { and } \forall \models \mathbf{I} \rightarrow \mathbf{A} \\
& \models \mathbf{E} \rightarrow \mathbf{O} \text { and } \not \models \mathbf{O} \rightarrow \mathbf{E} .
\end{aligned}
$$

(b) $\mathbf{E}$ and $\mathbf{I}$ are contradictories

(c) $\mathbf{A}$ and $\mathbf{E}$ are contraries

(d) $\mathbf{I}$ and $\mathbf{O}$ are subcontraries

(e) $\mathbf{A}$ and $\mathbf{I}$ are subalterns

(f) $\mathbf{E}$ and $\mathbf{O}$ are subalterns

To give an impression how the apparatus work I show the translation for each case and the corresponding E-valid reductions:

(a) $\models\left[\begin{array}{c}\forall x(F x \supset G x) \\ \exists x F x\end{array}\right] \rightarrow \neg\left[\begin{array}{c}\exists x(F x \wedge \sim G x) \\ \exists x F x\end{array}\right]$

$$
\left\{\begin{array}{c}
\vdash \forall x(F x \supset G x) \wedge \exists x F x \supset \sim \exists x(F x \wedge \sim G x) \\
\vdash \forall x(F x \supset G x) \wedge \exists x F x \supset \exists x F x
\end{array}\right\}
$$




$$
\begin{aligned}
& \models \neg\left[\begin{array}{c}
\exists x(F x \wedge \sim G x) \\
\exists x F x
\end{array}\right] \rightarrow\left[\begin{array}{c}
\forall x(F x \supset G x) \\
\exists x F x
\end{array}\right] \\
& \left\{\begin{array}{c}
\vdash \sim \exists x(F x \wedge \sim G x) \wedge \exists x F x \supset \forall x(F x \supset G x) \\
\vdash \sim \exists x(F x \wedge G x) \wedge \exists x F x \supset \exists x F x
\end{array}\right\}
\end{aligned}
$$

(b) $\models\left[\begin{array}{c}\forall x(F x \supset \sim G x) \\ \exists x F x\end{array}\right] \rightarrow \neg\left[\begin{array}{c}\exists x(F x \wedge G x) \\ \exists x F x\end{array}\right]$

$$
\begin{aligned}
& \left\{\begin{array}{c}
\vdash \forall x(F x \supset \sim G x) \wedge \exists x F x \supset \exists x(F x \wedge G x) \\
\vdash \forall x(F x \supset \sim G x) \wedge \exists x F x \supset \exists x F x
\end{array}\right\} \\
& \models \neg\left[\begin{array}{c}
\exists x(F x \wedge G x) \\
\exists x F x
\end{array}\right] \rightarrow\left[\begin{array}{c}
\forall x(F x \supset \sim G x) \\
\exists x F x
\end{array}\right] \\
& \left\{\begin{array}{c}
\vdash \sim \exists x(F x \wedge G x) \wedge \exists x F x \supset \forall x(F x \supset \sim G x) \\
\vdash \sim \exists x(F x \wedge G x) \wedge \exists x F x \supset \exists x F x
\end{array}\right\}
\end{aligned}
$$

(c) $\models\left[\begin{array}{c}\forall x(F x \supset G x) \\ \exists x F x\end{array}\right] \rightarrow \neg\left[\begin{array}{c}\forall x(F x \supset \sim G x) \\ \exists x F x\end{array}\right]$

$$
\left\{\begin{array}{c}
\vdash \forall x(F x \supset G x) \wedge \exists x F x \supset \sim \forall x(F x \supset \sim G x) \\
\vdash \forall x(F x \supset G x) \wedge \exists x F x \supset \exists x F x
\end{array}\right\}
$$

(d) $\models \neg\left[\begin{array}{c}\exists x(F x \wedge G x) \\ \exists x F x\end{array}\right] \rightarrow\left[\begin{array}{c}\exists x(F x \supset \sim G x) \\ \exists x F x\end{array}\right]$

$$
\left\{\begin{array}{c}
\vdash \sim \exists x(F x \wedge G x) \wedge \exists x F x \supset \exists x(F x \supset \sim G x) \\
\vdash \sim \exists x(F x \wedge G x) \wedge \exists x F x \supset \exists x F x
\end{array}\right\}
$$

(e) $\models\left[\begin{array}{c}\forall x(F x \supset G x) \\ \exists x F x\end{array}\right] \rightarrow\left[\begin{array}{c}\exists x(F x \wedge G x) \\ \exists x F x\end{array}\right]$

$$
\begin{gathered}
\left\{\begin{array}{c}
\vdash \forall x(F x \supset G x) \wedge \exists x F x \supset \exists x(F x \wedge G x) \\
\vdash \forall x(F x \supset G x) \wedge \exists x F x \supset \exists x F x
\end{array}\right\} \\
\forall \forall\left[\begin{array}{c}
\exists x(F x \wedge G x) \\
\exists x F x
\end{array}\right] \rightarrow\left[\begin{array}{c}
\forall x(F x \supset G x) \\
\exists x F x
\end{array}\right]
\end{gathered}
$$$$
\left\{\begin{array}{c}
\nvdash \exists x(F x \wedge G x) \wedge \exists x F x \supset \forall x(F x \supset G x) \\
\vdash \exists x(F x \wedge G x) \wedge \exists x F x \supset \exists x F x
\end{array}\right\}
$$

(f) $\models\left[\begin{array}{c}\forall x(F x \supset \sim G x) \\ \exists x F x\end{array}\right] \rightarrow\left[\begin{array}{c}\exists x(F x \wedge \sim G x) \\ \exists x F x\end{array}\right]$

$$
\begin{gathered}
\left\{\begin{array}{c}
\vdash \forall x(F x \supset \sim G x) \wedge \exists x F x \supset \exists x(F x \wedge \sim G x) \\
\vdash \forall x(F x \supset \sim G x) \wedge \exists x F x \ni x F x
\end{array}\right\} \\
\forall \forall\left[\begin{array}{c}
\exists x(F x \wedge \sim G x) \\
\exists x F x
\end{array}\right] \rightarrow\left[\begin{array}{c}
\forall x(F x \supset \sim G x) \\
\exists x F x
\end{array}\right]
\end{gathered}
$$




$$
\left\{\begin{array}{c}
\nvdash \exists x(F x \wedge \sim G x) \wedge \exists x F x \supset \forall x(F x \supset \sim G x) \\
\vdash \exists x(F x \wedge \sim G x) \wedge \exists x F x \supset \exists x F x
\end{array}\right\} .
$$

We observe that in regarding the second (presupposition) line we always get an elimination of conjunction. In (a) and (b) the same happens regarding the first (assertion) line. But to prove the assertion in (c) to (f) we necessarily need the existence premise. Unlike Leonard's strategy we now have a unified approach to the traditional square of opposites. It depends only on the innerlogical structure whether we have to use the represented existential presupposition.

\section{Outlook: Variable Quantifiers}

Let us first recall the reduction rules for quantifiers in $\mathcal{Q}$ :

$$
\begin{aligned}
& \forall \boldsymbol{x}\left[\begin{array}{l}
A \\
B
\end{array}\right] \Longrightarrow\left[\begin{array}{l}
\forall \boldsymbol{x} A \\
\forall \boldsymbol{x} B
\end{array}\right] \\
& \exists \boldsymbol{x}\left[\begin{array}{l}
A \\
B
\end{array}\right] \Longrightarrow\left[\begin{array}{l}
\exists \boldsymbol{x} A \\
\exists \boldsymbol{x} B
\end{array}\right]
\end{aligned}
$$

In this way these quantifiers do not yield the usual form of restricted classical quantifiers: $\forall x(F x \supset G x), \exists x(F x \wedge G x)$, due to

$$
\forall \forall x\left[\begin{array}{c}
G x \\
F x
\end{array}\right] \equiv \forall x(F x \supset G x) \quad \text { and } \quad \forall \exists x\left[\begin{array}{c}
G x \\
F x
\end{array}\right] \equiv \exists x(F x \wedge G x) .
$$

We can take an S5-like variable functor into consideration (cp. [5]):

$$
\square\left[\begin{array}{l}
A \\
B
\end{array}\right] \Longrightarrow\left[\begin{array}{l}
A \wedge B \\
A \wedge B
\end{array}\right] .
$$

This allows the simulation of an aspect of quantifying-in difficulties:

$$
\begin{aligned}
& \forall \exists x \square\left[\begin{array}{l}
G x \\
F x
\end{array}\right] \equiv \square \exists x\left[\begin{array}{c}
G x \\
F x
\end{array}\right] \quad \text { because of } \\
& \nvdash(\exists x F x \wedge \exists x G x) \supset \exists x(F x \wedge G x) .
\end{aligned}
$$

It is, of course, possible to introduce variable quantifiers which act like classical quantifiers:

$$
\begin{aligned}
& \forall_{1} \boldsymbol{x}\left[\begin{array}{l}
A \\
B
\end{array}\right] \Longrightarrow\left[\begin{array}{l}
\forall \boldsymbol{x}(B \supset A) \\
\forall \boldsymbol{x}(B \supset A)
\end{array}\right] \\
& \exists_{1} \boldsymbol{x}\left[\begin{array}{l}
A \\
B
\end{array}\right] \Longrightarrow\left[\begin{array}{l}
\exists \boldsymbol{x}(B \wedge A) \\
\exists \boldsymbol{x}(B \wedge A)
\end{array}\right] .
\end{aligned}
$$


Then, it holds that

$$
\models \forall_{1} x\left[\begin{array}{c}
G x \\
F x
\end{array}\right] \equiv \forall x(F x \supset G x) \text { and } \models \exists_{1} x\left[\begin{array}{c}
G x \\
F x
\end{array}\right] \equiv \exists x(F x \wedge G x) \text {. }
$$

Despite the logical equivalence between an E-subformula and a CL-subformula we have to make a clear distinction between the classical/external negation (a functor) and the presupposition-preserving negation (a variable functor):

$$
\begin{array}{ll}
\models \sim \forall_{1} x \neg\left[\begin{array}{c}
G x \\
F x
\end{array}\right] \equiv \exists x(F x \wedge G x) & \models \sim \forall_{1} x \neg\left[\begin{array}{c}
G x \\
F x
\end{array}\right] \equiv \exists_{1} x\left[\begin{array}{c}
G x \\
F x
\end{array}\right] \\
\models \sim \exists_{1} x \neg\left[\begin{array}{c}
G x \\
F x
\end{array}\right] \equiv \forall x(F x \supset G x) & \models \sim \exists_{1} x \neg\left[\begin{array}{l}
G x \\
F x
\end{array}\right] \equiv \forall_{1} x\left[\begin{array}{c}
G x \\
F x
\end{array}\right]
\end{array}
$$

Last but not least, it is worth noting that a little modification of the system $\mathcal{Q}$ gives the possibility of introducing mixed variable quantifiers:

$$
\begin{aligned}
& \forall_{\exists} \boldsymbol{x}\left[\begin{array}{l}
A \\
B
\end{array}\right] \Longrightarrow\left[\begin{array}{l}
\forall \boldsymbol{x}(B \supset A) \\
\exists \boldsymbol{x}(B \wedge A)
\end{array}\right] \\
& \exists_{\forall} \boldsymbol{x}\left[\begin{array}{l}
A \\
B
\end{array}\right] \Longrightarrow\left[\begin{array}{l}
\exists \boldsymbol{x}(B \wedge A) \\
\forall \boldsymbol{x}(B \supset A)
\end{array}\right]
\end{aligned}
$$

It is a matter of further research whether special variable quantifiers can be interpreted as generalized quantifiers.

\section{References}

[1] Frege G., "Über Sinn und Bedeutung", Zeitschrift für Philosophie und philosophische Kritik, NF 100 (1892), 25-50.

[2] Hailperin T., "Quantification theory and empty individual domains", The Journal of Symbolic Logic 18 (1953), 197-200.

[3] Leblanc H., Meyer R. K., "Open formulas and the empty domain", Zeitschrift für mathematische Logik und Grundlagen der Mathematik 12 (1969), 78-84.

[4] Leonard H. S., "The logic of existence", Philosophical Studies 7 (1956), 49-64.

[5] Max I., "Zur Explikation der Modaloperatoren von S5 mittels G-Funktorenvariablen", [in:] Logik in der Semantik - Semantik in der Logik, ed. E. Dölling, Berlin 1987, 123-133. 
[6] Mostowski A., "On the rules of proof in the pure functional calculus of the first order", The Journal of Symbolic Logic 16 (1951), 107-111.

[7] Quine W. V. O., "Quantification and the empty domain", The Journal of Symbolic Logic 19 (1954), 177-179.

[8] Strawson P. F., Introduction to Logical Theory, London 1952.

INGOLF MAX

Department of Philosophy

Friedrich-Schiller-University

Zwätzengasse 9

D-07740 Jena, Germany

e-mail: xim@.rz.uni-jena.de 